\title{
Image Inpainting - An Inclusive Review of the Underlying Algorithm and Comparative Study of the Associated Techniques
}

\author{
Mahroosh Banday \\ Post Graduate Scholar \\ Department of Electronics and Communication, DIT \\ Dehradun
}

\author{
Richa Sharma \\ Assistant Professor \\ Department of Electronics and Communication, \\ DIT Dehradun
}

\begin{abstract}
Image In-painting, the technique that aims to revert deterioration (scratches, artifacts in photographs and videos) in images in an undetectable form, is as ancient as artistic creation itself. Digital Image In painting, a relatively young research area is an art of filling in the missing or corrupted regions in an image using information from the neighbouring pixels in a visually plausible manner, while restoring its unity. In painting which is essentially an image interpolation problem has numerous applications. It is helpfully used for object removal in digital photographs, image reconstruction, text removal, video restoration, special effects in movies disocclusion and so on. Several approaches have been proposed by the researchers to correct the occlusion. This proposed work presents a comparative study to provide a comprehensive visualization of different image in painting techniques. In this paper different types of image in painting algorithms are placed in juxtaposition. The algorithms are analysed theoretically as well as experimentally, based on which a ranking of algorithms will be established over different kinds of applications in diverse areas .
\end{abstract}

\section{General Terms}

In-painting, Exemplar, Hybrid, Texture Synthesis

\section{Keywords}

Inpainting, Texture, Structure, Image, Occlusion, Object Removal, Algorithm, Exemplar

\section{INTRODUCTION}

Image In-painting has received considerable attention in the past few years and has become a very active field of research in image processing. The concept of image inpainting commonly known as disocclusion or image completion originates from the medieval art practices, the motive being to recreate the lost or damaged structures in order to bring the medieval art up to date so as to make the damaged artwork more discernible, while conserving its unicity[4],[8]. The need to interpolate the image in an undetectable manner naturally extended from painting to photography and videos in an effort to automate many tedious artefact correction methods on images and videos.
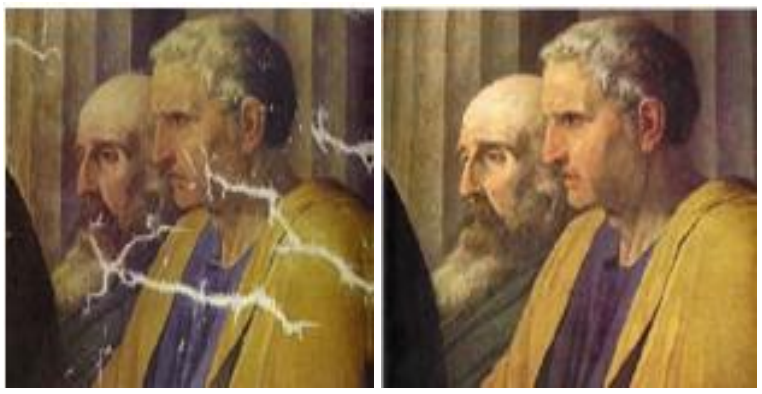

Fig.1: Manual Inpainting performed by a professional artist.

Figure 1 shows the manual in-painting done by artists for centuries to retouch the oil paintings in a way that renders the changes made in the painting as imperceptible as possible. The in-painting problem first appeared in digital domain under the name 'error concealment' in telecommunications where the need was to recreate lost or damaged image structures / blocks that have been lost during data transmission and coding (e .g streaming video) in order to make it more legible. The main goal of Digital Image Inpainting is to reconstruct the lost or deteriorated parts of an image or videos utilizing spatial information from its neighbouring areas in order to bring the damaged paintings or old pictures close to the original or back to the original state so that it seems natural to the human eye [12]. Digital image in-painting can be thought of as a linear or non-linear transformation [30] as shown in figure 2 , where $I_{0}$ is the original image and I is the digitally in painted /transformed image.

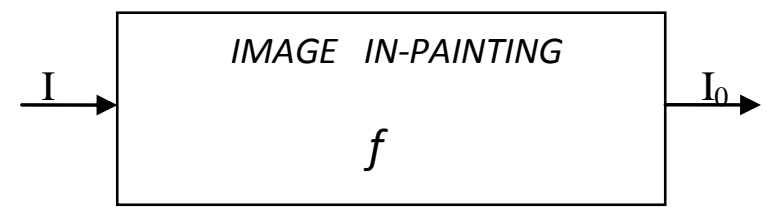

Fig.2: Linear transformation of Image $I_{0}$ through an Image Processor f

Digital Image In-painting has found widespread use in numerous applications such as image restoration, error recovery of images and videos, object removal, restoration of image data lost during transmission, text removal from images, digital zooming, red eye correction and special effects in movies. Natural images being composite, image information may be divided into three parts i.e,.shape/structure, texture and colour information. The 
structure inpainting usually focuses on the continuity of the geometrical structure of an image. Textures can either be homogenous (repeated texels) or stochastic (random texels).The texture to be synthesized is learned from a texture sample from the known part of the image. A number of algorithms for image inpainting continuously emerge due to extensive research and technology advancements, the basic idea of all being to improve the general quality of an image and to achieve better performance in terms of quality of the in painted images.

The In-painting Model :

Image I can be mathematically represented as:

$$
\mathrm{I}=\left\{\begin{array}{l}
\Omega \subset R_{e}^{n} \rightarrow R_{e}^{m} \\
X \rightarrow I(X)
\end{array}\right.
$$

Here, $x$ is a vector representing spatial coordinates of a pixel defined as $X=(x, y)$ in case of a $2 D$ image $(n=2)$. In case of a colour image, each pixel is composed of three colour components $(m=3)$ i.e., $R, G, B$ in colour space[25].The region to be in painted is assumed to have gone through a deterioration represented by operator $\mathrm{D}$, which removed information from the image. The image $I$ is defined as a union of the source region ' $\mathrm{S}_{\mathrm{I}}$ ' (known part of the image) and the unknown part of image, $\mathrm{U}_{\mathrm{I}}(\Omega)$ i.e. $\mathrm{I}=\mathrm{S}_{\mathrm{I}} \cup \mathrm{U}_{\mathrm{I}}$. Thus the degraded image $\mathrm{I}_{\mathrm{D}}$ can be expressed as $\mathrm{I}_{\mathrm{D}}=\mathrm{DI}$.

The main aim of inpainting is thus to restore images with partly information loss. The first step of inpainting is to manually select the portion of the image that needs to be restored. After this, image reconstruction is done automatically by estimating the pixels in the unknown region $\mathrm{U}_{\mathrm{I}}$ (figure3) from the pixels located in the source region $\mathrm{S}$ to revert deterioration making it look reasonable to the casual observer.

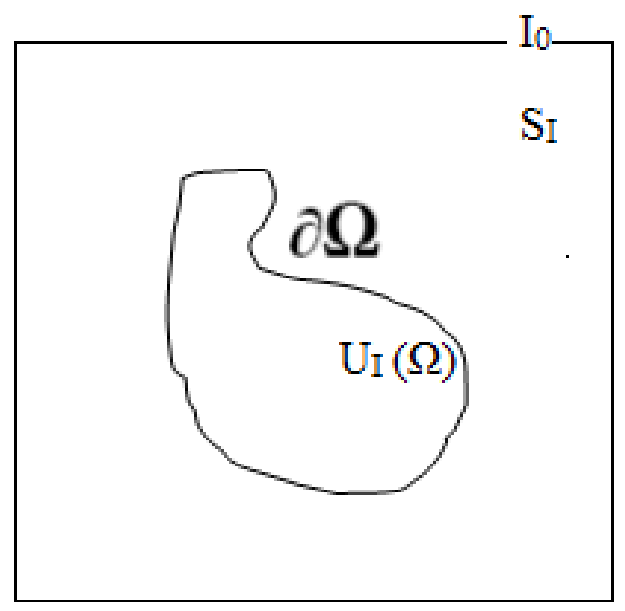

Fig.3: The image I0, the region $\Omega$ to be in-painted and its boundary $\partial \Omega$.

The general in painting algorithm can be described as:

Step I: Specify the missing region $\Omega$.

Step II: Specify the boundary ( $\partial \Omega$ ) of the corrupted

area.
Step III: Initialize the missing region $(\partial \Omega)$.

Step IV: In-paint all the pixels (x, y) $€ \Omega$ based on the information found on the hole's edge $(\partial \Omega)$.

Paper Outline: The next section of this paper presents various digital image inpainting techniques and their experimental results which include PDE based inpainting, Texture synthesis based inpainting, Exemplar based inpainting, Hybrid inpainting and Fast Digital in-painting and Convolution based Method. Results from various techniques are analysed and compared in Section III. Various applications of in-painting techniques are discussed in Section IV. Finally, concluding remarks based on experimental results obtained from both real scene photographs and synthetic images are presented in section V.

\section{IMAGE IN PAINTING TECHNIQUES}

The most challenging chore in image in painting technique is the evaluation of the quality of the image so that the in painted image seems reasonable to the human observer. In painting in general can be viewed as a set of image reconstruction techniques. Various approaches have been used by the researchers for digital image in painting which may be classified into the following broad categories:

- Partial Differential Equation (PDE) based In painting.

- Texture Synthesis based In painting.

- Exemplar based In painting.

- Hybrid In painting.

- Fast Digital In painting and Convolution Based Method.

\subsection{Partial Differential Equation (PDE) based In painting:}

The first digital inpainting technique was the diffusion based in-painting. In this technique the corrupted or the missing region is filled by diffusing information from the surrounding areas into the missing region at pixel level in a way that the changes seem undetectable to the observer.

Isotropic Diffusion stems from the linear heat flow equation:

$$
\left\{\begin{array}{l}
I_{t=0}=I_{D} \\
\frac{\partial I}{\partial t}=\Delta I
\end{array}\right.
$$

where $I_{D}$ is the deteriorated version of the original image I and $\Delta \mathrm{I}$ is the image laplacian. The time variable ' $\mathrm{t}$ ' denotes the progression of the function $I$ in a sequential continuous manner. Isotropic diffusion which minimises the variations acts as a low pass filter that suppresses high frequencies in an image. And it is because of this reason that isotropic method suffers from blur close to the contours. Thus more general methods using non linear Partial Differential Equations that were earlier used for fluid dynamics have been introduced to preserve sharpness and edges thereby increasing the efficiency of this method. Figure 4 shows the in-painted output of the damaged image using PDE technique. 


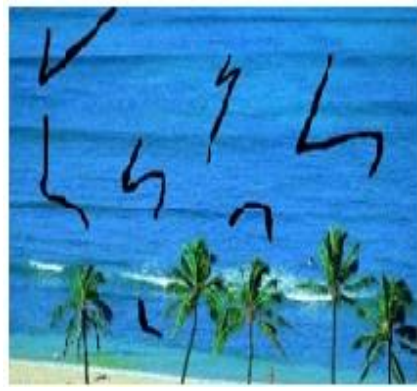

(a)

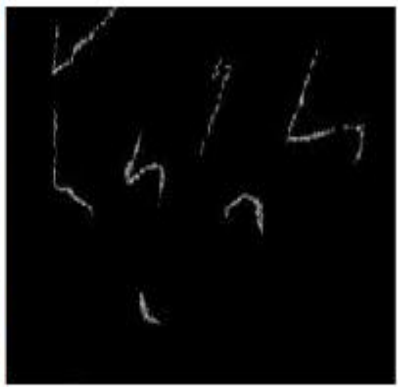

(b)

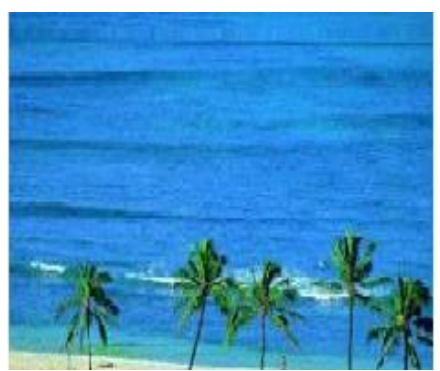

(c)

Fig.4: In-painted results of PDE based image in-painting (a) The Original Image , (b) The mask, (c) The Result.

Bertalmio et.al [17] have proposed a digital image inpainting algorithm based on Partial Differential Equation (PDE) in which the direction of the lines of equal luminescence i.e. isophotes (figure 5) is maintained by evaluating the direction of the largest spatial change obtained by computing a gradient vector and rotating this vector by 90 radians. Bertalmio et.al took the ideas from computational fluid dynamics (CFD) to continue the isophote lines into the region to be in painted [16]. It treats the image intensity as a stream function and the laplacian of the image as vorticity of the fluid which is continued into the region to be in painted by vector fields defined by stream function .PDE based algorithm is an iterative algorithm. The basic idea behind the algorithm is to propagate both the geometric information (gradient direction) and the photometric information (gray-scale values) of the image that is available at the boundary of the occluded area ( $\partial \Omega$ ) into the area to be filled in $(\Omega)$. This is done by formulating a partial differential equation which propagates the information (laplacian of image) in the direction of minimal change using 'isophote lines' (the lines of equal gray value) [21],[31].This method is thus based directly on the Navier Strokes Equations for CFD. The PDE technique for in painting produces good results if the region to be filled in is small but if the missed regions are large ,the efficiency of this algorithm decreases as it takes a long time to fill in large missing areas and the results produced will also be unsatisfactory.

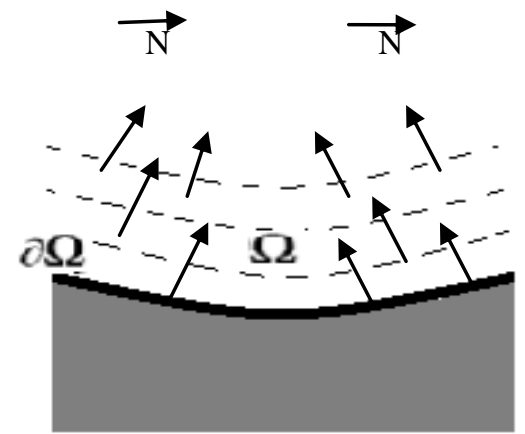

Fig.5: Direction of the lines of equal gray value (isophote lines).

Inspired by the work of Bertalmio at.al, Chan \& Shen forth put Total Variational (TV) in painting algorithm based on second order partial differential equation [20],[26],[29]. This algorithm uses Euler- Lagrange model as well as anisotropic diffusion based on the intensity of the isophotes. This algorithm performs quite well for noise removal applications and for filling in small regions. But the weak point of this model is that it neither greats texture patterns nor connects the broken edges. The TV model was then extended to the Curvature Driven Model (CDM) which also included the geometric information of the isophotes in order to handle the curved structures in a better way thus allowing this inpainting technique to proceed over large areas [28]. Although CDM connects the broken edges but also results in some blur. Then Telea [27] proposed a Fast Marching Method (FMM) which in-paints the near pixels to known areas first and also maintains a narrow band of pixels which distinguishes the unknown pixels from the known pixels. The drawback of this method is that it produces a post inpainting blur which becomes noticeable when the area to be in painted is larger than ten pixels. This method computes the smoothness of an image and is considered as a PDE method which is simpler as well as faster to implement as compared to other PDE based techniques. PDE being an iterative method is very time consuming, requires difficult implementation process and is not applicable to large textured areas, the results for which are often blocky. PDE based technique has numerous applications such as restoration, image segmentation etc. Partial Differential Equation (PDE) based algorithms focuses on maintaining the geometric structure of the area to be in painted.

\subsection{Texture Synthesis Based Inpainting:}

PDE based techniques are well suited for filling in small gaps, text overlays etc but PDE technique usually fails if applied to areas containing regular patterns or to a textured area. This failure is because of the following reason:

1 .Mostly high intensity gradients are present in textures which may be interpreted wrongly as edges and falsely transported into the region to $\mathrm{b}$ in painted.

2. In case of PDE based in painting, the information used is only the boundary condition that is present within a narrow band around the region to be in painted. Thus, it is impossible to recognize structures, textured areas or regular patterns from such an insignificant amount of information.

Texture synthesis based algorithms are based on the concept of synthesising artificial texture from an (typically small) initial seed, striving to preserve the appearance (structure and colour) in a way that the synthesized texture matches perfectly with the sample texture [15],[24]. It 
synthesis an arbitrary sized texture to complete the missing regions by the help of similar neighbourhoods of the corrupted or the missing pixels as depicted in figure 6. Early in painting approaches employed these methods for filling in the corrupted regions by first sampling and then copying pixels from the surrounding neighbourhood. For example, in [3] Markov Random Field (MRF) is utilized for modelling the local pixel distribution after which a new texture is created by inquiring existing texture and retrieving information from similar neighbourhoods. Their difference mainly lies in the way continuity is maintained between the already existing pixels and the in painting domain (hole). This pixel based texture synthesis performs very well on set of selected images. Synthesis based approaches are well suited for only a selected set of images where completing the missing region with homogenous textures results in nature completion. Since the filling- in of the in painting domain is done pixel by pixel, this scheme is very slow.

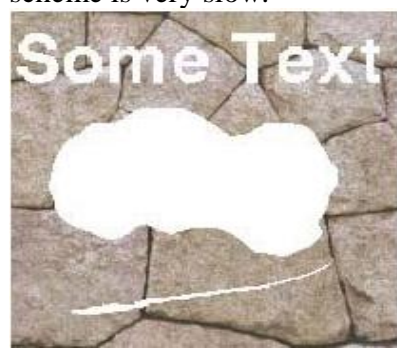

(a)Input Image

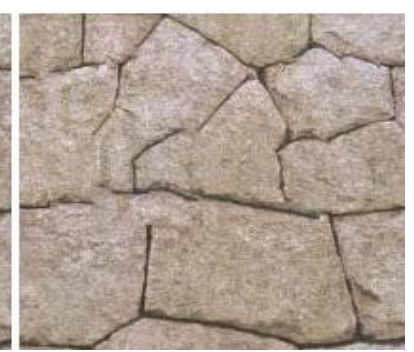

(b)Texture Synthesis
Fig.6: Example of texture synthesis (a) Input Corrupted Image, (b) In-painted output using texture synthesis.

Efros \& Freeman proposed a way to greatly improve the speed by using more simpler fragment based algorithm. Fragment based algorithms can be considers ad generalized texture synthesis. Here, instead of copying pixels one by one entire blocks are transferred into the in painting hole that results in smoothness and the changes in the in painted area seem undetectable to the Human Visual System (HVS).

Criminisi et.al [1], introduced an algorithm specific for texture in painting that uses the same technique as proposed by EfrosLeung [3], the only difference being that the high priority is given to the filling of pixels placed along the edges. Priority of a pixel is dependent on the gradient magnitude of its surrounding areas and the confidence term. Pixels lying close to the edges have high gradients and thus edges are given high priority. This slight modification was sufficient to give even better results as depicted in [1]. Later, this technique was extended to fast synthesising algorithm. It works by stitching small patches of the images together referred to as image quilting.

Multiresolution texture synthesis approach which can generate texture in varying brightness conditions was proposed by Yamauchi et.al [9]. Later, this technique was extended to fast Multiresolution texture synthesis. A fast Multiresolution image completion method based on texture synthesis was introduced by Fang et.al [5]. In this method texture matching inside the hole can be accelerated through Vector Quantization (VQ) and Principal Component Analysis (PCA) based methods.

Innumerable texture synthesis methods for creating textures with different statistical characteristics are available other than fore cited but here we shall confine ourselves only to those synthesis techniques specifically used for in-painting .Texture synthesis techniques can be categorized into three broad categories:
1. Statistical (i.e parametric) Texture Synthesis Technique.

2. Pixel based (i.e non parametric) Texture Synthesis Technique.

3. Patch based (i.e non parametric) Texture Synthesis Technique.

Statistical texture synthesis methods are only able to produce irregular/stochastic textures but fail to produce regular/structured textures .The fill-in in the pixel based in painting method is done pixel by pixel irrespective of applying filters on it. The results produced by pixel based method are of better quality than those produced by statistical /parametric methods. Patch based methods as opposed to pixel based methods 'build on' a sample texture patch by patch (figure7), yielding visually plausible and faster texture in painting.
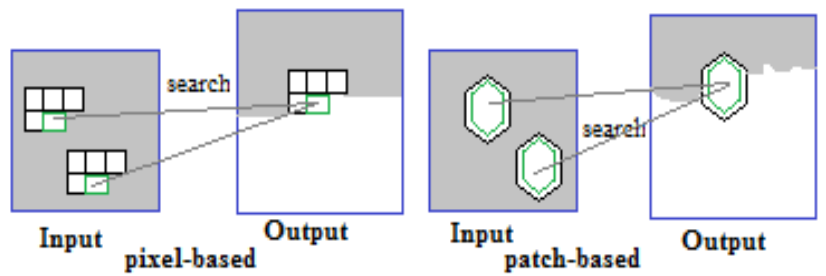

Fig.7: Difference between pixel-based and patch-based Texture Synthesis.

Unlike pixel based algorithm where the copy is just a copy, in patch passed algorithms the issue is more complicated .The patch being larger than a pixel overlaps with the already synthesized areas for which some decision is to be made as to how these conflicting areas can be handled .Texture synthesis based in painting techniques specifically perform well in approximating textures but found difficulty with natural images as they contain geometric structures in the form of edges and have complicated interactions between texture and structure boundaries . Sometimes, this technique also requires the user to specify which to be replaced and the place to be replaced.

A recent study on patch based texture synthesis methods has stated that special purpose algorithms are required to develop for handling different types of textures. Keeping this thing into consideration, it can be concluded that no universal texture synthesis algorithm is present and it still remains an aim to desire. Although the texture synthesis is esteemed in in-painting, it is sagacious to understand that these techniques address only a small range of in painting issues and thus are not well suited for a wide range of applications in diverse areas .Texture synthesis techniques could be employed for repairing digitized photographs and if the damaged area needs to be filled-in with some regular pattern, texture synthesis does a good job.

\subsection{Exemplar Based In painting:}

As natural images (paintings) are composed of both structures and textures (regions with regular patterns), more complex in painting techniques are required for faithfully reconstructing the corrupted regions. Because of this distinguishing feature of natural images, a technique that is designed strictly for texture synthesis will not provide satisfactory results. Exemplar based in painting inspired by local region growing techniques that grow texture using one patch or one pixel at a time perform well for a wide range of images and are able to produce reasonably better quality results by combining texture synthesis with isophote (lines of equal gray value) driven in painting methods. Exemplar based methods have proved to be 
very effective for reconstructing large target regions. The basic idea behind Exemplar based image interpolation is the use of a set of image exemplars/ blocks that are derived either from the same image that needs to be in painted or from other images that are related to the representative images. Exemplar based approach consists of two basic steps:

Firstly, priority assignment is done. Each pixel p belonging to the patch $\psi p$ has a patch priority given by the product of the confidence term $\mathrm{C}(\mathrm{p})$ and the data term $\mathrm{D}(\mathrm{p})$. The confidence term tells us about the number of existing pixels in this patch. The data term tells us about the strength of the isophote that hits the boundary and is important since it maintains the structure of an edge at the target patch and focuses on the linear structure to be synthesized first.

Mathematically patch priority is given as:

$$
P(p)=C(p) D(p)
$$

where,

$$
C(p)=\frac{\sum_{q \in \Psi p \cap \Phi} C(q)}{|\Psi p|}
$$

and

$$
D(p)=\frac{\left|\nabla^{\perp} I_{n p} \cdot \overrightarrow{n p}\right|}{\alpha}
$$

where, $|\Psi p|$ is the area of $\Psi p, n p$ is normal to the front $\partial \Omega, \nabla^{\perp} I_{n p}$ is the isophote located at $\mathrm{p}$ and $\alpha$ is the normalization factor that is equal to 255 for an 8 bit gray-scale image.

Secondly, this approach synthesizes the region to be in painted iteratively by selecting most similar patch whose similarity is measured by some metrics from the known region of the image.

In general, exemplar based in painting algorithm can be described as follows:

Firstly, initialize the confidence term $C(p)$ assigning $C(p)=1$ for $p \varepsilon \phi$ and $C(p)=0$ for $p \varepsilon \Omega$. The below given steps are then repeated unless the fill front $\partial \Omega^{t}=\Phi$

1. First the fill front $\partial \Omega$ is identified.

2. The patch priorities of all those patches whose centres align on fill front $\partial \Omega$ are computed.

3. The patch $\Psi p$ having the maximum patch priority is chosen.

4. The best matching candidate patch $\Psi q$ is chosen from the source region $\Phi$ using SSD cost function .The SSD is used to calculate the colour distance between the source patch $\Psi q$ and the target patch $\Psi p$.

$$
\Psi q=\arg \min _{\Psi q \subset \Phi} d(\Psi q, \Psi p)
$$

$$
\begin{gathered}
\text { where, } d(\Psi q, \Psi p)=\sum_{(i, j)}\left|\boldsymbol{I}\left(\boldsymbol{P}_{(i, j)}\right)-\boldsymbol{I}\left(\boldsymbol{q}_{(i, j)}\right)\right|^{2}, \\
\forall\left(p_{(i, j)} \in \Psi p\right) \quad \text { and } \forall\left(q_{(i, j)} \in \Psi q\right)
\end{gathered}
$$

5. Update the information from $\Psi q$ to $\psi p$. In this step, the boundary $\partial \Omega$ of the target region $\Omega$ and the desired information for evaluating filling priorities are updated, which may contribute to the minimization of blocky effect (artefacts).

6. Update the confidence term of the pixel $p$ in the region to be in painted, $\forall p \varepsilon(\Psi p \cap \Omega)$. The confidence value decays as the filling proceeds indicating that we are not sure about the colour value of the pixels towards the centre of the region to be in painted.

\subsubsection{Basic Exemplar Based In painting Methods:}

A number of algorithms have been proposed for exemplar based image in painting. Jia et.al [11] presented a technique based on texture segmentation .This technique segments an image into many regions on the basis of its texture and colour features, thereby in painting each region separately thus producing smooth linking of structures across hole in addition to propagating textural information. This algorithm requires an expensive segmentation process and also finds it hard to decide as to what constitutes boundary between two textures. In order to overcome this drawback, Drori [10] introduced a fragment based in painting method that iteratively searched, approximated and updated details by compositing fragments. But the drawback of this algorithm is that its in painting time is intolerable. Bertalmio [18] proposed a hybrid algorithm that combined the diffusion based/isophote driven in painting and texture synthesis.
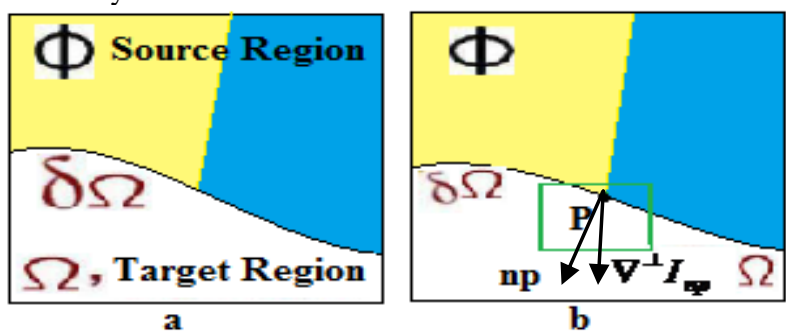

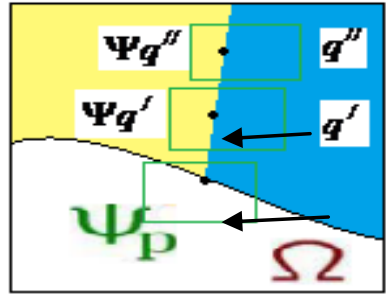

c

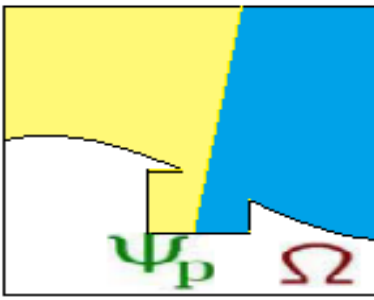

d
Fig.8:Criminisi et.al Exemplar based Texture synthesis Algorithm.(a)Original image with target region $(\Omega)$, its source region $\phi$ and boundary d $\Omega$. (b) Area to be synthesized delimited by the patch $\psi \mathrm{p}$ centred at point p.(c) Most likely candidate patches $(\psi q /, \psi q / /$ lying in the source region $\phi$. (d)Best matching patch among candidate patches copied to the position occupied by $\psi p$, thereby achieving partial fill-in in target region $\Omega$. 
Discussing the exemplar based in painting methods, one of the techniques frequently given as a reference is the one introduced by Criminisi et.al [1], [2], an approach that has been designed to encourage fill-in from the boundaries of target region where the strength of the nearby pixels is strong. Figure 8 illustrates this point in a simpler way. This algorithm has been designed for filling in large gaps with visually plausible information from the surroundings areas. This algorithm uses the method of SSD i.e Sum of Squared Differences to choose the best matching patch among the candidate patches in the source region and then uses the priority based mechanism for determining the region filling order. Exemplar based algorithm successfully fills in large gaps preserving the linear image structure. For example in figure 8 , the information from the top right corner of the patch $p$ is used for finding similar candidate patches from the known part of the image. The image quality depends greatly on the order in which the filling of patches is done. According to this approach, the pixels that lie on the edge path are given high priority and are filled earlier than rest of the pixels that lie on the boundary of the area to be in painted[2],[23]. After finding the highest priority pixel $p_{\text {belonging to the patch }}$ $\psi \mathrm{p}$, this method searches for the best matching patch among the candidate patches $\psi \mathrm{q} /, \psi \mathrm{qq} / /$ in the known region of the image, $\phi$ using SSD cost function. The best matching patch among the candidate patches is thus used to fill in the high priority patch $\psi \mathrm{p}$ centred around the pixel $\mathrm{p}$. Other important aspects of this approach that determine the quality of the image is the size of the patches. The size of the patches must be chosen carefully. There is no method to automatically adjust the patch size so it is usually done manually. This is actually the weak point of this algorithm as smaller patches result in a noticeable pattern as well as increased in painting time and large patches increase the chances of choosing a bad patch containing undesirable information. Another weak point of this method is that it gives unsatisfactory results in case the target region is spread along most of the image area that reduces the number of candidate patches required for filling in the corrupted regions. However, for a wide range of images, this algorithm gives impressive results unlike PDE and texture synthesis based methods that inevitably produce blur in the region that has been in painted.

\subsubsection{Improved Exemplar Based In painting Method that is based on Local Optimization:}

An improved version of the method proposed by Criminisi et.al was introduced by Zhou \& Kelly in [13]. This algorithm uses the same priority based mechanism but instead of choosing the candidate patches on the basis of similarity to the patch that is to be filled-in, Zhou \& Kelly devised an algorithm that preserves the local consistency of the target region by selecting the optimal patch that gives the maximum probability of matching with the patch $\Psi p$ to be filled in. This algorithm gives good results than those given by Criminisi et.al's technique. This method avoids over smoothing thereby extending and preserving structural and textural details of images.

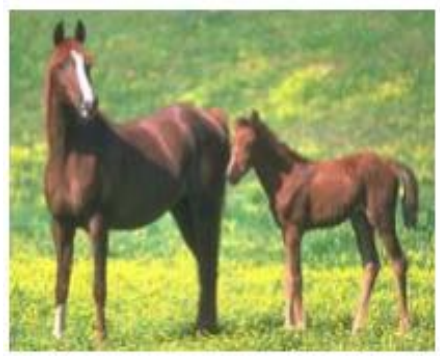

(a)

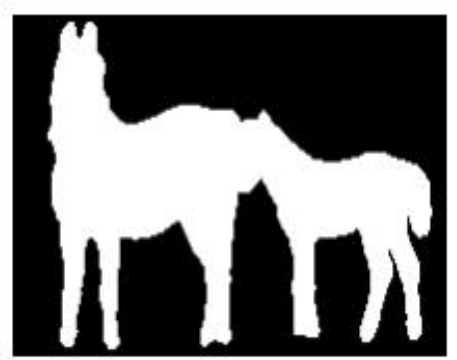

(b)

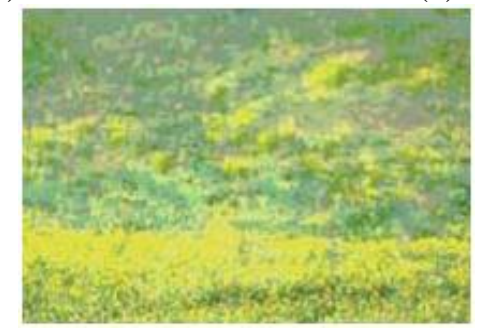

(c)

Fig. 9: Removing Large Objects using Exemplar based Texture Synthesis (a) Original Image, (b) Developed Mask , (c) In-painted Result .

Exemplar based in painting methods produce good results if and only if the missing / corrupted regions comprise of simple texture and structure as illustrated in figure 9 but if the samples in the image are not enough to synthesize the target region, it is impossible to achieve satisfactory results using this approach. However, exemplar based algorithms introduce good quality patch matching scheme which blends both the structure and texture of the image, thereby producing results that appear reasonable to the casual observer. Fast exemplar algorithms are projected to speed up the computation efficiency thereby reducing the in painting time.

\subsection{Hybrid Inpainting}

Partial differential equation based methods perform well for small and sparingly distributed gaps. They are suitable for sparse smooth images and for disseminating strong structures but PDE based methods are unable to synthesize textures. On the other hand, exemplar based in painting performs well in synthesizing textures with regular/homogenous patterns. Unlike PDE they do not preserve the linear structure of images with small holes spread over most of the image area.

Natural images are composed of composite structures and textures. The structures comprise of primary sketches (corners, edges) of an image and textures are regions with regular/ homogenous feature statistics. A technique is thus required to handle complex images containing both structures and textures. Hybrid image in painting technique mostly used for filling large missing regions preserve both the texture and the structure of an image so that the changes in the in painted area are undetectable to the human eye. The basic idea behind the hybrid in painting technique is that it decomposes the image into two components, texture and structure regions [6],[15] as depicted in figure 10. 


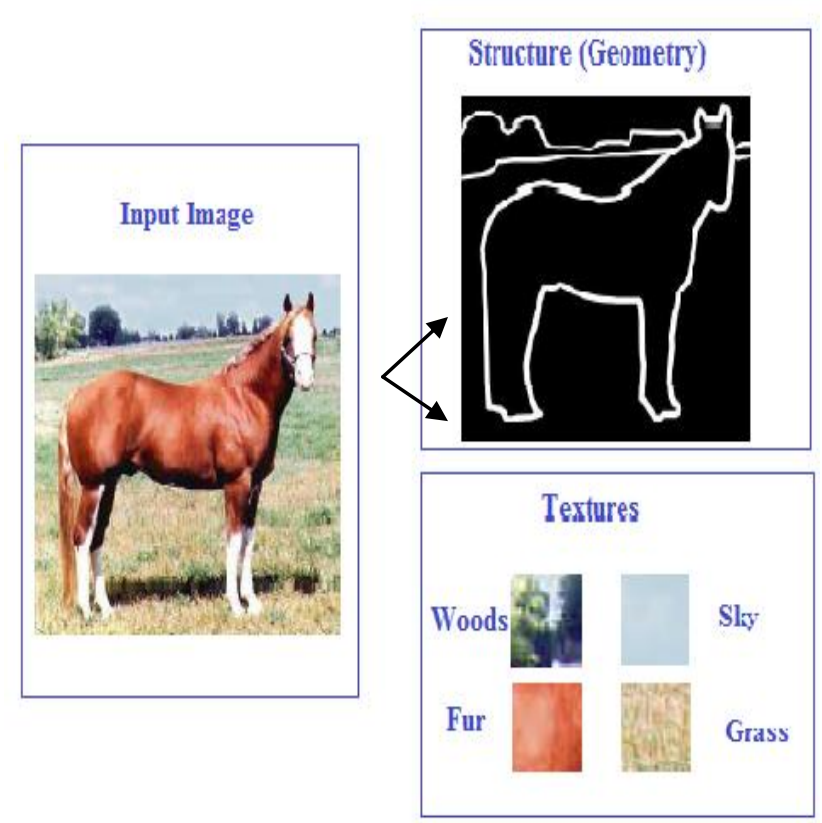

Fig. 10: Decomposition of an image into Structural (geometric) and textural components.

The corresponding decomposed regions are then in painted separately with diffusion based isophote driven in painting algorithms and exemplar-based methods, whichever suits best. Jiying $\mathrm{Wu}$ et.al [14] proposed a hybrid in painting model where the texture and the structure can be separated automatically using a total variational (TV) method. Here the image is decomposed as a sum of two different functions, one representing the texture and second one representing an image structure. The texture component is in painted using exemplar based texture synthesis method and geometric structure is in painted using diffusion based method. The structure image is an approximation of primary sketches in an image containing only edges which separate the smooth regions. These sparingly smooth images are also known as the cartoon images [22].The two in painting functions are then summed up to produce the final in painted result. After this, K.A.Narayanakutliet.al proposed hybrid image in painting algorithm for occlusion removal. This technique is unsupervised decomposition method and is completely data driven that splits image into mode functions (intrinsic) and residue by both texture synthesis and structure driven in painting methods. J.Stark et.al proposed a new hybrid method that is based on sparse representation for splitting an image into geometric and texture component, called layers using two dictionaries of different characteristic features $I g \& I t$. Thus, the decomposed image is a sum of structural and textural part as $I=I g v g+I t v t$. This approach is known as MCA i.e, Morphological Component Analysis. These two dictionaries, $I g \& I t$ are mutually coherent, each dictionary giving a sparse representation for one part while yielding a non sparse representation for the another part. These dictionaries are then combined into a big dictionary which is used by a pursuit algorithm to determine the sparse representation of each component. The sparse vectors for the two layers are obtained by $\min \|v g\|_{p}+\|v t\|_{p}$ where, $\|\bullet\|$ denotes $L p$ norm with $p$ equal to either zero or one. A penalization term is then added to solve the minimization problem. It regularizes sparse approximation of the image. The hybrid technique fills in the target region with composite structures and textural patterns. This technique however, introduces blocky effect when the missing region is large. The novel diffusion technique restores the smoothness \& preserves the linear structure of an image and the texture part is synthesized by exemplar based method that is constrained by a cross-isophote diffusion term. Theoretical hypothesis and experimental results have proved that hybrid image in painting model can in paint both structure and texture image components well.

\subsection{Fast Digital In painting and Convolution based Method:}

Depending on the size of the gap that needs to be filled in, all the in painting techniques discussed earlier require minutes to hours for an in painting algorithm to complete to fully in paint the missing regions, thereby making it inefficient for interactive user applications. Thus, a new class of fast in painting techniques are developed to speed up the conventional in painting techniques. A.Telea introduced a fast marching algorithm almost similar to PDE technique but considerably simple and fast to implement than other PDE methods. This algorithm computes smoothness of an image from already known neighbourhood of an image as a weighted sum to in paint the gap. The limitation of this approach is that it produces blur when the region to be in painted is thicker than ten pixels. After this, Oliveira et.al (2001) introduced a fast, iterative image in painting convolution based method[19].

This algorithm consists of the following (figure 11) four steps:

1. Select the corrupted or the missing area $(\Omega)$ to be in painted manually.

2. Detect the boundary ( $\partial \Omega$ ) of the region to be in painted.

3. Initialize the missing region $(\Omega)$ by clearing its colour information.

4. Convolve the region to be in painted with the weighted average kernel repeatedly.

This algorithm makes use of one of the following weighted kernels, having a zero weight at the kernel's centre:

$$
\left[\begin{array}{lll}
a & b & a \\
b & 0 & b \\
a & b & a
\end{array}\right] \quad\left[\begin{array}{lll}
c & c & c \\
c & 0 & c \\
c & c & c
\end{array}\right]
$$

where, $\mathrm{a}=0.073235, \mathrm{~b}=0.176765, \mathrm{c}=0.125$.

This algorithm stops when no more pixels are left whose values are varied by more than a particular threshold as a result of earlier iterations. The plus point of this algorithm is that it is faster than other image in painting algorithms and is well suited for images that don't have high frequency components or high contrast edges. This algorithm produces the output faster in few seconds with both the diffusion templates after more than hundred (100) iterations but produces blurring if the region to be in painted is large. The strength of this algorithm is represented by its simplicity which makes it easier and faster to implement and understand. Mohiy and Moustafa[22] modified the Oliveira In painting Model to reduce the restoration time and remove the blurring 
effect. This Modified Convolution based in painting algorithm reduced the number of iterations from 100 to one iteration and it could also remove large objects from symmetric background images, thereby increasing the quality of results. The basic difference is in the method of filling and this algorithm has modified the diffusion kernels as:

$$
\left[\begin{array}{lll}
a & b & a \\
b & a & b \\
a & b & 0
\end{array}\right] \quad\left[\begin{array}{lll}
c & c & c \\
c & c & c \\
c & c & 0
\end{array}\right]
$$

where, $\mathrm{a}=0.073235, \mathrm{~b}=0.176765, \mathrm{c}=0.125$.

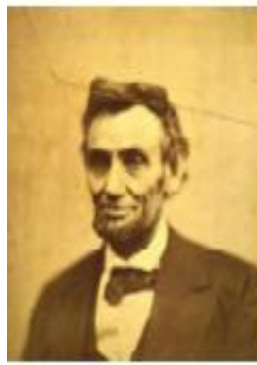

$$
\text { D }
$$

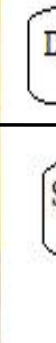

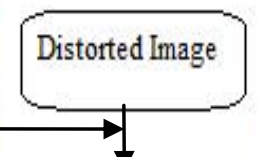
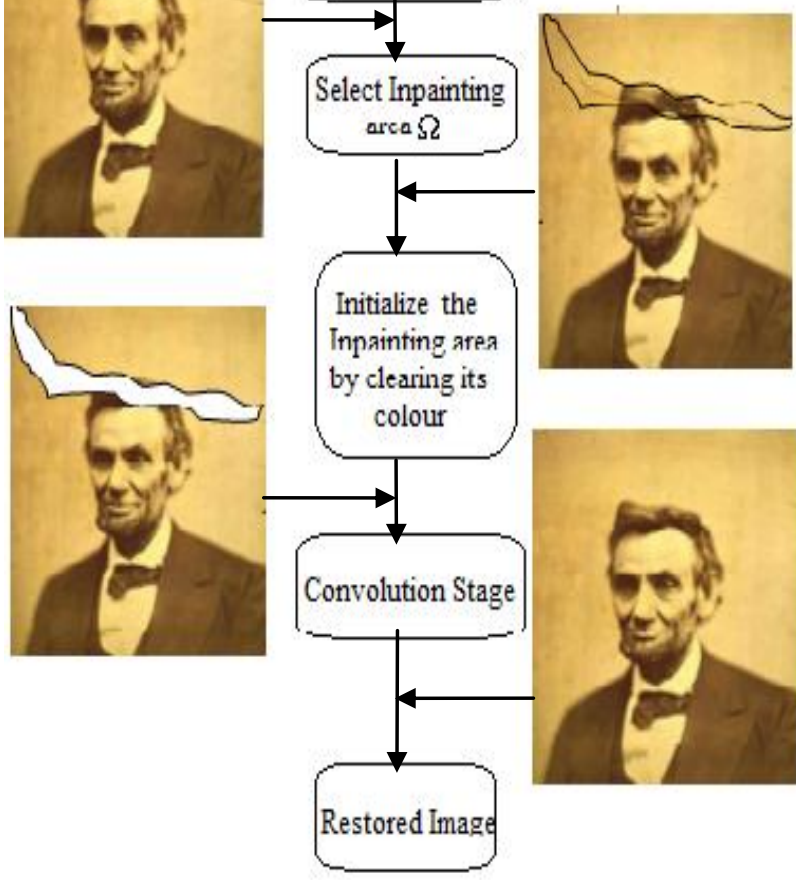

Fig.11: Block Diagram of Convolution based Image Inpainting Method.

The region to be in painted is selected and convolved with the averaging filter (kernel) having zero weight at the bottom right corner unlike the previous one having zero weight at the centre. This modification proscribes the need to iterate the convolution as the goal of repetition is achieved from the first iteration only. This fast convolution based digital in painting algorithm is faster than Oliveira's in painting algorithm with one iteration only rather than hundred (100). This algorithm also removes large objects from symmetric background images and that too without blurring but fails to produce good results while removing large objects from natural images.

\section{RESULTS, ANALYSIS AND DISCUSSION}

Digital image in-painting has become a hotspot and has received a lot of attention since a decade .Several approaches have been introduced with varying applicability in disocclusion, texture synthesis, object removal, text removal, and image reconstruction. These techniques are based on various approaches and include basic as well as improved and more recent methods to fill-in the corrupted regions in a visually plausible manner so that the in-painted area mimics the source region in appearance.

Table1 shows the results of various in-painting techniques. Diffusion based methods perform well if the area to be inpainted is uniform. The performance is high for noise removal applications and when the area to be in-painted is small. But the drawback of this method is that it neither connects the broken edges nor synthesizes texture, the results for which often produce blur. Also, PDE being an iterative method is very time consuming. Texture synthesis based methods are well suited for only a selected set of images where completing the missing region with homogenous textures results in nature completion. This technique performs well for in-painting large areas but founds difficulty with natural images as they contain geometric structures (edges). Exemplar based methods faithfully reconstruct both the structure and the texture and give impressive results for a wide range of images as it combines texture synthesis with diffusion driven methods. The disadvantage of this method is that it gives unsatisfactory results if the patch size is small and if the target region is spread along most of the image area. Hybrid In-painting approach restores the smoothness and preserves the linear structure as well as the texture of an image. This technique is well suited for images with simple structure and textures but produces blur when the missing region is large and the patch size is inappropriate. Fast digital in-painting Convolution based method is a new class of in-painting techniques that enhance the speed of in-painting manyfold. This technique removes large images from symmetric backgrounds but gives unsatisfactory results while removing objects from natural images. Table 2 sheds light upon the merits and the demerits of different in-painting approaches. Based on the varying applicability of different in-painting techniques, all the approaches are equally significant in diverse areas of application. 
TABLE 1 : Results of various Inpainting Techniques

\begin{tabular}{|c|c|c|}
\hline $\begin{array}{c}\text { Inpainting } \\
\text { Methods }\end{array}$ & Original Image & In-painted Image \\
\hline $\begin{array}{l}\text { PDE } \\
\text { Inpainting } \\
\text { Technique }\end{array}$ & & \\
\hline $\begin{array}{l}\text { Texture } \\
\text { Synthesis }\end{array}$ & & \\
\hline $\begin{array}{l}\text { Exemplar } \\
\text { based } \\
\text { Texture } \\
\text { Synthesis }\end{array}$ & & \\
\hline $\begin{array}{l}\text { Hybrid } \\
\text { Inpainting }\end{array}$ & & \\
\hline $\begin{array}{l}\text { Modified } \\
\text { Convolution } \\
\text { based } \\
\text { Inpainting }\end{array}$ & & \\
\hline
\end{tabular}

Table 2 A comparison of image Inpainting methods

\begin{tabular}{|c|c|c|}
\hline METHODS & MERITS & DEMERITS \\
\hline $\begin{array}{l}\text { Partial differential } \\
\text { Equation (PDE) }\end{array}$ & $\begin{array}{l}\text { Fine Outcome } \\
\& \text { preserves } \\
\text { All Structural } \\
\text { information }\end{array}$ & $\begin{array}{l}\text { Results display } \\
\text { blurring artifacts } \\
\text { when applied to } \\
\text { large missing } \\
\text { regions }\end{array}$ \\
\hline $\begin{array}{l}\text { Total variation } \\
\text { In-painting } \\
\text { (TV) }\end{array}$ & $\begin{array}{l}\text { Works well for } \\
\text { removing salt } \\
\text { and pepper } \\
\text { noise }\end{array}$ & $\begin{array}{l}\text { Models Inpainting } \\
\text { only in small/ } \\
\text { miniature regions }\end{array}$ \\
\hline $\begin{array}{l}\text { The curvature } \\
\text { Driven Diffusion } \\
\text { Model (CDD) }\end{array}$ & $\begin{array}{l}\text { Used for bigger } \\
\text { areas }\end{array}$ & $\begin{array}{l}\text { Connects only } \\
\text { few broken edges }\end{array}$ \\
\hline $\begin{array}{l}\text { Texture Synthesis } \\
\text { based In-painting }\end{array}$ & $\begin{array}{l}\text { Results do not } \\
\text { display blur/ } \\
\text { artifacts }\end{array}$ & $\begin{array}{l}\text { Not applicable for } \\
\text { thick scratched } \\
\text { regions \& curved } \\
\text { structures }\end{array}$ \\
\hline $\begin{array}{c}\text { Pixel Based } \\
\text { Texture Synthesis }\end{array}$ & $\begin{array}{l}\text { Mimics both } \\
\text { deterministic } \\
\text { and stochastic } \\
\text { textures }\end{array}$ & $\begin{array}{l}\text { Requires a lot of } \\
\text { time as whole } \\
\text { source sample } \\
\text { area is required to } \\
\text { be checked }\end{array}$ \\
\hline $\begin{array}{c}\text { Patch Based } \\
\text { Texture Synthesis }\end{array}$ & $\begin{array}{c}\text { Better } \\
\text { performance, } \\
\text { applicable to } \\
\text { large area, } \\
\text { faster }\end{array}$ & $\begin{array}{l}\text { Produces blur in } \\
\text { case of } \\
\text { inappropriate } \\
\text { patch size }\end{array}$ \\
\hline $\begin{array}{l}\text { Exemplar based } \\
\text { Texture Synthesis }\end{array}$ & $\begin{array}{l}\text { Gives } \\
\text { impressive } \\
\text { results and } \\
\text { preserves all } \\
\text { structural \& } \\
\quad \text { textural } \\
\text { information }\end{array}$ & $\begin{array}{c}\text { Gives } \\
\text { unsatisfactory } \\
\text { results if the } \\
\text { corrupted region } \\
\text { is spread along } \\
\text { most of the image } \\
\text { area }\end{array}$ \\
\hline $\begin{array}{l}\text { Hybrid In- } \\
\text { painting }\end{array}$ & $\begin{array}{l}\text { Restores } \\
\text { smoothness and } \\
\text { preserves both } \\
\text { the linear } \\
\text { structure and } \\
\text { texture of Image }\end{array}$ & $\begin{array}{c}\text { Produces blocky } \\
\text { effect } \\
\text { if the damaged } \\
\text { area is large \& the } \\
\text { patch size is } \\
\text { inappropriate }\end{array}$ \\
\hline $\begin{array}{l}\text { Convolution } \\
\text { Based In-painting } \\
\text { Method }\end{array}$ & $\begin{array}{l}\text { Produces fine } \\
\text { results without } \\
\text { blurring }\end{array}$ & $\begin{array}{l}\text { For corrupted } \\
\text { region thicker } \\
\text { than ten pixels, } \\
\text { blurring occurs }\end{array}$ \\
\hline
\end{tabular}




\begin{tabular}{|c|c|c|}
\hline Modified & Enhances the & Gives poor results \\
Convolution & speed of & while removing \\
Based In-painting & Inpainting & large objects from \\
& manifold, & natural images \\
& Reduces & \\
& number of & \\
& iterations from & \\
100 to 1 & \\
\hline
\end{tabular}

\section{APPLICATIONS}

The purpose of image in-painting is to restore the unicity of the picture by reconstructing the missing parts of the image in a way that the changes made seem undetectable to the casual observer. The art of in-painting plays a crucial role in many image processing applications: disocclusion, red eye removal, image reconstruction, loss concealment, image resizing, special effects in movies, text removal and so on. We have illustrated some main applications of in-painting techniques showing limited applicability of some of them in particular cases.

IMAGE RESTORATION:-Image in-painting deals with the reconstruction/restoration of the corrupted or damaged images. It removes degradations like text overlays, scratches $\&$ cracks in old photographs, dust spots in films, stamped data from photographs and red eye removal. Another important application is the finger-print restoration in the automatic finger-print identification systems [7]. Since the missing regions are generally not too large in the restoration problem, hence PDE based and patch based methods give satisfactory results.

OBJECT REMOVAL:-Another important application of image in-painting is to remove foreground objects from an image leaving a hole to be filled-in. The local diffusion method introduces blurring artefacts and hence is unfit for this application. Hence, in order to recover the texture of the hole, exemplar- based methods and hybrid methods work well for this type of application.

DISOCCLUSION:-Disoccluded areas are the missing regions spread along the image area. Disocclusion results in pixels with the unknown colour value. Thus the value of these pixels needs to be determined using the in-painting approach so as to make the image more captivating. Hybrid in-painting and the Curvature Driven Diffusion (CDD) methods are well suited for this kind of application.

LOSS-CONCEALMENT:-Image and video transmissions usually suffer from packet losses which results in missing regions in the output image that appears after decoding.The loss concealment is the next step after decoding to recover the blocks that had been lost during transmission/coding of images as in a streaming video. The in-painting approaches are useful in this context as well to reconstruct the lost parts of an image by taking advantage of the dependencies within the image or between the frames/adjacent images in a video sequence. As far as this application is concerned, the patch based methods using exemplars as well as the hybrid methods combining exemplar-based technique and diffusion method give good results.

\section{CONCLUSIONS}

Image in-painting is a relatively young and active research area. This study provides estimable contextual information about different types of image in-painting techniques with an aim to revert deterioration and to add or remove elements from an image/video in a visually plausible manner. Digital in-painting approaches can be used for all kinds of image repairs such as disocclusion, erasing objects from an image, text removal, repairing scratches \& cracks and so on. The success of in-painting techniques is quantified in a precise way by the quality assessment of how well the geometric (structure) and the photometric (colour) information is propagated into the target region. After surveying different methods, it is observed that each in-painting technique presented here has a number of advantages as well as shortcomings. It is observed that PDE based in-painting algorithms are used for structure dominated images to fill-in narrow and small scratched and cracked regions, but can't fill in large missing regions while the texture synthesis performs well for textured images only. Theoretical analysis proves that exemplar based texture synthesis produces better results for in-painting both the structure as well as textured images, provided the missing region consists of simple texture and structure. Fast and convolution based in-painting methods have enhanced the speed of in-painting manifold but are only suited for images having symmetric backgrounds. One has to rely on human visual comparisons (HVC) to qualitatively evaluate whether the in-painted results are physically credible and visually captivating.

\section{OPEN PROBLEMS}

Despite extensive research carried out in the field of image inpainting, the problem of in-painting is still far from being completely solved as many challenges lay ahead in making inpainting robust and practical in day to day applications. Here, we have highlighted some of the possible research areas which can merit future investigations to extend the art of inpainting.

(i) Extension to 3-D:- From the last few years, attempts have been made to extend the in-painting techniques from two dimensions (2-D) to three dimensions(3-D). This modified methodology would help in the restoration of historical artefacts and damaged monuments. This is because the objects that are symmetrical in three dimensions (3-D)may not appear symmetric in their 2-D projection / image plane. However, extensive research is still needed in this direction for results to be visually pleasing.

(ii) In-painting for high resolution images:Reconstruction/In-painting of high resolution images is still an issue of concern . Dealing with high resolution images, main issue for the in-painting techniques is a significant increase in the computation time (upto several hours). Thus to quantify the quality of in-painting techniques, new algorithms can be developed that would be able to inpaint high-resolution images.

(iii) Video In-painting:-Video In-painting still remains a challenging and a crucial task as the arbitrary camera motion, tracking moving objects in a video, and variability in illumination conditions remain difficult problems to be solved and hence complicate the in-painting performance. Another issue in video in-painting is to preserve the visual coherency of the in-painted results over time.

(iv) Reducing In-painting time:- More efficient algorithms are required to be developed for reducing the computational cost and In-painting time.

(v) Quality Evaluation:-Accessing the quality of the inpainted images is another open problem as no such quality metrics exists that does not depend upon the reference image. 
So one has to rely on the human visual comparisons for the quality assessment.

\section{REFERENCES}

[1] Criminisi, A., P'erez, P. and Toyama, K. (2003). Object Removal by Exemplar-Based Inpainting, Proceedings of the 2003, IEEE Computer Society Conference on Computer Vision and Pattern Recognition, pp:1-8.

[2] Criminisi,A.., Perez, P. and Toyama, K. (Sep 2004). Region Filling and Object Removal by Exemplar based Image In-Painting, IEEE Trans. on

Image Processing, 13, pp: 1200-1212.

[3] Efors, A. and Leung, T.K. (1999). Texture synthesis by non-parametric sampling, Proceedings of the 17th IEEE International Confere on Computer Vision, pp: 10331038.

[4] Alexandra Ioana Oncu Feier. (2012). Digital Inpainting for Artwork Restoration: Algorithms and Evaluation, Master Thesis Report.

[5] Fang C.W and Lien,J.J.J. Fast image replacement using multi-resolution approach

[6] Christine Guillemot and Olivier Le Meur.2014. Image Inpainting- Overview and recent advances, IEEE signal processing magazine,pp:127-144,ISSN: 1053-5888.

[7] Bornemann, F. and Marz,T. (July 2007). Fast image inpainting based on coherence transport, J. Math. Imaging Vis,28(3), pp:259-278.

[8] Emile-Male,G. (1976). The Restorer's Handbook of Easel Painting, Van Nostrand Reinhold, New York.

[9] Yamauchi,H., Haber,J., Seidel, H.P. (2003). Image Restoration Using Multiresolution Texture Synthesis and Image Inpainting, Computer Graphics International, pp:108-113.

[10] Drori,I., Cohen-Or,D. and Yeshurun,D. (2003). Fragment-based image completion, ACM Transactions on Graphics, 22, pp: 303-312.

[11] Jia,J. and Tang,C.K. (2003). Image repairing: Robust image synthesis by adaptive and tensor voting, Proceedings of IEEE Computer Society Conference on Computer Vision Pattern Recognition, pp: 643-650.

[12] Shen,J. (June 2003). Inpainting and the Fundamental Problem of Image Processing, SIAM News, 36(5).

[13] Zhou,J. and .Kelly,A.R.(2010). Image inpainting based on local optimization, International Conference on Patteren Recongnition (ICPR).

[14] Jiying Wu, Qiuqi Ruan. (May, 2008). A Novel Hybrid Image

in-painting" IEEE Transactions on Image Processing.

[15] Komal Mahajan, Prof. Vaidya,B.M.(2012). Image in Painting Techniques: A survey, IOSR Journal of Computer Engineering ,5(4),pp:45-49.

[16] Bertalmio,M., Bertozzi,A.L. and Sapiro,G. (Dec 2001). Navier stokes, Fluid Dynamics, and Image and Video Inpainting, Proceedings of Conf. Comp.Vision Pattern Rec., Hawai, pp:355-362.
[17] Bertalmio,M., Sapiro,G., Caselles,V., and Ballester,C. (July 2000). Image Inpainting, Proceedings of SIGGRAPH, New Orleans, USA, pp: 417-424.

[18] Bertalmio,M., Vese,L., Sapiro,G., and Osher,S. (2003). Simultaneous Structure and Texture Image Inpainting, Proceedings of IEEE conference on Computer Vision and Pattern Recognition.

[19] Oliveira, M., Bowen, B., McKenna, R., Chang,Y. (2001) .Fast Digital Inpainting, Proceedings of the International Conference on Visualization, Imaging and Image Processing, Marbella, Spain, pp: 261-266.

[20] Mahalingam, Vijay Venkatesh. (2010). Digital Inpainting Algorithms and Evaluation, University of Kentucky,Doctoral Dissertations. Paper 55, http://uknowledge.uky.edu/gradschool_diss/55

[21] Michael E Taschler. (2006). A Comparative Analysis of Image Inpainting Techniques, The University of York, pp:01-120.

[22] Mohiy M. Hadhoud, Kamel. A. Moustafa and Sameh. Z. Shenoda. ( 2006). Digital Images Inpainting using Modified Convolution Based Method, International Journal of Signal Processing, Image Processing and Pattern Recognition.

[23] Neelima1, N., Arulvan,M.. (2013). Object Removal by Region Based Filling Inpainting, IEEE ,978-1-46735301-4.

[24] Nirali Pandya and Bhailal Limbasiya. (December 2013) A Survey on Image Inpainting Techniques , International Journal of Current Engineering and Technology, 3(5),pp: $1828-1831$

[25] Gonzales,R.C., and Woods,R.E. (2002). Digital Image Processing, Second Edition, Prentice Hall, ISBN: 0-20118075-8.

[26] Cham,T., and Shen,J. (2001). Local inpainting models and TV inpainting, SIAM Journal on Applied Mathematics,62,pp:1019-1043

[27] Telea. (2004). An Image Inpainting Techniques Based On The Fast Maching Method,Journal Of Graphics Tools,9(1).

[28] Chan, T.F., and Shen, J. ( September 2000). Non-Texture Inpainting by Curvature Driven Diffusion (CDD), UCLA Computational and Applied Mathematics ,pp:00-35.

[29] Chan,T.F., Shen, J.,and Vese, L. ( Dec. 2002). Variational PDE Models in Image Processing, UCLA Computational and Applied Mathematics , pp:02-61.

[30] Yasmine Nader El-Glaly. (2007). A Thesis on Development of PDE-based Digital Inpainting Algorithm Applied to Missing Data in Digital Images.

[31] Zhongyu Xu, Xiaoli Lian and Lili Feng. (2008). Image Inpainting Algorithm Based on Partial Differential Equation, IEEE Computer Society,International Colloquium on Computing, Communication, Control, and Management,ISSN:978-0-7695-3290-5. 